\title{
Measurements of Excision Repair Tracts Formed during Meiotic Recombination in Saccharomyces cerevisiae
}

\author{
PETER DETLOFF ${ }^{1,2}$ AND THOMAS D. PETES ${ }^{1 *}$ \\ Department of Biology, University of North Carolina, Chapel Hill, North Carolina 27599-3280, ${ }^{1}$ and Department \\ of Molecular Genetics and Cell Biology, University of Chicago, Chicago, Illinois $60637^{2}$
}

Received 23 October 1991/Accepted 18 January 1992

\begin{abstract}
During meiotic recombination in the yeast Saccharomyces cerevisiae, heteroduplexes are formed at a high frequency between HIS4 genes located on homologous chromosomes. Using mutant alleles of the HIS4 gene that result in poorly repaired mismatches in heteroduplex DNA, we find that heteroduplexes often span a distance of $1.8 \mathrm{~kb}$. In addition, we show that about one-third of the repair tracts initiated at well-repaired mismatches extend $900 \mathrm{bp}$.
\end{abstract}

During meiosis in Saccharomyces cerevisiae, heterozygous alleles $A$ and $a$ usually segregate $2 A: 2 a$ into the four spores of the ascus. The two most common types of aberrant segregation are gene conversion events ( $3 A: 1 a$ or $1 A: 3 a$ segregation) and postmeiotic segregation (PMS events). For a heterozygous auxotrophic mutation, PMS events are signalled by sectored colonies formed when the spore colonies grown on rich medium are replica plated to omission medium. For most mutant alleles in yeast cells, PMS events are much rarer than gene conversion events (median frequencies of 0.5 and $5 \%$ of unselected tetrads, respectively [12]). The most common types of PMS tetrads in $S$. cerevisiae are those with $2 A: 1 a: 1 A / a$-sectored colony or $1 A: 2 a: 1 A / a$-sectored colony $(12,28)$. Since comparable patterns of PMS were first detected in eight-spored fungi (in which meiotic divisions are followed by a single mitotic division), these types of segregation are described as $5 A: 3 a$ or $3 A: 5 a$ tetrads, respectively. In order to use a single system of nomenclature, we describe gene conversion events as $6+: 2 \mathrm{~m}$ or $2+: 6 \mathrm{~m}$ and normal Mendelian segregation as $4+: 4 \mathrm{~m}$.

Sectored spore colonies reflect the presence of heteroduplex DNA with an unrepaired base mismatch (10). Since, in $S$. cerevisiae, tetrads with a single PMS event $(5+: 3 \mathrm{~m}$ or $3+: 5 \mathrm{~m})$ are usually much more common than tetrads with two PMS events (aberrant $4+: 4 \mathrm{~m}$ ), most heteroduplexes are thought to be the result of the nonreciprocal transfer of a single strand of DNA from one allele to the other $(12,28)$. For example, if one strand of DNA is donated from the $A$ allele to the $a$ allele, one would expect to get a $5 A: 3 a$ pattern of segregation.

Although all PMS events are thought to represent unrepaired mismatches in heteroduplexes, two different models of gene conversion have been proposed. In one class of models, the conversion events are the result of repair of mismatches in heteroduplex DNA $(17,25,31)$. Such repair events may involve excision of the mismatch and surrounding sequences from one strand of the duplex followed by repair of the resulting gap, using the other strand as the template for DNA synthesis. In the original version of the double-strand break repair model (38), it was proposed that most conversion events reflect the repair of a doublestranded gap with sequences derived from the homologous chromatid. Several lines of evidence indicate that most

\footnotetext{
* Corresponding author.
}

meiotic conversion in $S$. cerevisiae is the result of mismatch repair. First, DNA mismatch repair mutants of $S$. cerevisiae that increase the frequency of PMS while decreasing the frequency of gene conversion have been isolated (42). Second, the frequency of PMS per aberrant segregation depends on the particular base pair mismatch $(5,21,41)$.

The length of meiotic gene conversion tracts in $S$. cerevisiae has been estimated in several studies. In studies of coconversion events at the $A R G 4$ locus, Fogel and Mortimer (11) estimated that the typical gene conversion tract spanned a few hundred base pairs. Conversion tracts of greater genetic distance (sometimes greater than 14 centimorgans) were found by DiCaprio and Hastings (7) at the SUP6 locus. In experiments in which the physical distance between markers was known, the length of an average conversion tract was estimated to be 1.5 to $3.7 \mathrm{~kb}(4,19,37)$.

The length of a gene conversion tract is influenced by two factors: the length of the heteroduplex region and the length of the excision repair tract. The length of the heteroduplex region can be examined by measuring the frequency of co-PMS events for markers with defined physical map positions. Although co-PMS events have been detected in several studies in $S$. cerevisiae $(7,12)$, these events are usually infrequent because most of the mutant alleles used in these studies showed little PMS. In addition, the physical distances separating the markers were not known for most combinations of alleles. One exception to this generalization is an analysis of coconversion and co-PMS in strains heterozygous for the arg4-16 and arg 4-17 mutations, located 214 bp apart (41). Of about 3,000 tetrads, 21 showed co-PMS for these markers and 190 showed coconversion (12).

The amount of DNA excised and resynthesized during mismatch repair (an excision repair tract) has been investigated in most detail in bacteria (26). One method of measuring such tracts is to transform cells with heteroduplexes containing multiple mismatches. Using this procedure, Wagner and Meselson (39) showed that the average length of a repair tract in Escherichia coli was $3 \mathrm{~kb}$. Transformation studies in Streptococcus pneumoniae suggested that repair tracts in this organism range from 5 to $10 \mathrm{~kb}(14,24)$. A cocorrection study in $S$. cerevisiae suggests that repair tracts on plasmids transformed into vegetatively dividing haploids are usually shorter than $1 \mathrm{~kb}(2)$.

A method of determining the length of meiotic excision tracts is to examine the effects of alleles that lead to 
well-repaired mismatches (low-PMS alleles) on the PMS frequency of nearby alleles that lead to poorly repaired mismatches (high-PMS alleles). The rationale for this approach is that high-PMS alleles may represent mismatches that are poorly recognized by the mismatch repair system; if a mutant allele that leads to a well-recognized mismatch is inserted nearby, the excision repair tract initiated at this allele may sometimes include the poorly recognized mismatch, leading to a reduction in PMS and an elevation in gene conversion $(12,16,32)$. This result has been obtained in a number of studies. For example, the PMS rate of arg4-16 is reduced from $48 \%$ of the aberrant segregations to about $28 \%$ in the presence of arg4-17 (upstream of arg4-16) or arg4-19 (downstream of arg4-16) (13). Since the arg4-16 and arg 4-17 mutations are 214 bp apart (41), this result indicates that the excision repair tract spans $200 \mathrm{bp}$ about half of the time. The PMS rate of his $1-49$ was reduced from $85 \%$ of the aberrant segregations of $18 \%$ in the presence of the heterozygous his 1-1S allele (15); the his 1-1S allele by itself shows no PMS. In both studies, although the PMS level of the high-PMS allele was reduced, the level of PMS for the low-PMS allele was not elevated.

In quantitative studies of the length of the excision repair as measured by the technique described in the preceding paragraph, it is important to determine how often heteroduplexes include both mismatches. If heteroduplexes include only one of the two mismatches, an excision tract derived from that mismatch would have no opportunity to affect the gene conversion frequency of the other allele. One method of determining the length of heteroduplex within a gene is to analyze the co-PMS frequencies of mutant alleles that have high levels of PMS. We chose to examine heteroduplex formation and mismatch repair at the HIS4 locus for several reasons. First, in the strain background that we use, mutations at the HIS4 locus have an extraordinarily high level of aberrant segregation (approximately 10 -fold higher than normal) $(5,28)$. Second, most of the aberrant segregations at this locus have a heteroduplex intermediate (28). Third, the HIS4 gene and many mutant derivatives have been sequenced $(1,8,9,29)$. Using strains that contain one or more of these sequenced mutations, we show that (i) many of the heteroduplexes at the HIS4 locus span alleles located near the $5^{\prime}$ and $3^{\prime}$ ends of the gene $(1.8 \mathrm{~kb})$ and (ii) almost all excision repair tracts are longer than $26 \mathrm{bp}$ and about one-third span interallelic distances of $900 \mathrm{bp}$.

\section{MATERIALS AND METHODS}

Plasmids and $E$. coli strains. Plasmids phis4-519 (B137), phis4-712 (B201), phis4-713 (B138), and phis4- 229 (B136) were gifts from B. Ruskin and G. Fink. These plasmids contain mutant his 4 genes on an EcoRI-BamHI fragment inserted into EcoRI-Bam HI sites of YIp5. The his 4 mutations in these plasmids were sequenced previously (9) and are shown in Table 1. The his4-3133 allele was constructed by inserting the self-annealed palindromic oligonucleotide (5'-CTAGTTACTGTATGTACATACAGTAA) into the $X b a I$ site (located near the $3^{\prime}$ end of HIS4) of pMW2. The pMW2 plasmid contains a $B g l I I$ fragment with the $3^{\prime}$ end of HIS4 inserted into the BamHI site of YIp5 (a gift of M. White). The plasmid resulting from the insertion of the palindrome into pMW2 is pPD6. The site of insertion was confirmed by DNA sequence analysis using methods described by Kraft et al. (20).

E. coli DK1 $[\Delta($ srl-recA $) 306$ araD139 $\Delta($ ara leu $) 7697$ $\Delta$ lacX74 galU galK hsdR strA mcrA mcrB (22)] was used to
TABLE 1. Related haploid yeast strains constructed by the two-step transplacement procedure

\begin{tabular}{|c|c|c|c|c|}
\hline $\begin{array}{l}\text { Strain } \\
\text { name }\end{array}$ & $\begin{array}{l}\text { Untrans- } \\
\text { formed } \\
\text { strain }\end{array}$ & Plasmid & $\begin{array}{l}\text { Relevant } \\
\text { genotype }^{a}\end{array}$ & $\begin{array}{l}\text { Position (type) } \\
\text { of mutation }^{b}\end{array}$ \\
\hline PD7 & AS4 & phis4-519 & his4-519 & +493 (insertion of a G) \\
\hline PD22 & AS13 & phis4-712 & his 4-712 & +1396 (insertion of a G) \\
\hline PD25 & AS4 & phis4-712 & his4-712 & +1396 (insertion of a G) \\
\hline PD23 & AS4 & phis4-713 & his4-713 & +2270 (insertion of a G) \\
\hline PD24 & AS13 & phis4-713 & his4-713 & +2270 (insertion of a G) \\
\hline DNY47 ${ }^{c}$ & AS13 & pDN22 & his4-IR9 & $\begin{array}{l}+467 \text { (18-bp palindromic } \\
\text { insertion) }\end{array}$ \\
\hline PD21 & AS13 & phis4- $\Delta 29$ & his $4-\Delta 29$ & -87 to +938 (deletion) \\
\hline PD68 & AS4 & phis4- $\Delta 29$ & his4- 429 & -87 to +938 (deletion) \\
\hline \multirow[t]{2}{*}{ PD100 } & DNY47 & pPD6 & his4-IR9 & $\begin{array}{l}+467 \text { (18-bp palindromic } \\
\text { insertion) }\end{array}$ \\
\hline & & & his4-3133 & $\begin{array}{l}+2327 \text { (26-bp palindromic } \\
\text { insertion) }\end{array}$ \\
\hline
\end{tabular}

a Mutational changes made within the HIS4 region.

$b$ The +1 position represents the first base pair in the initiating ATG of HIS4.

${ }^{c}$ Strain constructed previously (27).

propagate plasmid pPD6. E. coli DB6507 [hsd $S 20\left(\mathrm{r}_{\mathrm{B}}{ }^{-} \mathrm{m}_{\mathrm{B}}{ }^{-}\right)$ supE44 ara14 $\lambda$ - galK2 lacY1 proA2 rspL20 xyl-5 mtl-1 rec $A 13$ mcr $A(+) m c r B(-)$ tyrS $\left.74:: \operatorname{Tn} 5 \mathrm{Km}^{\mathrm{r}} \operatorname{str} A\right]$ was used to propagate plasmids phis4-519, phis4-712, phis4-713, and phis4- $\Delta 29$.

Yeast strains. All yeast strains used in this study were derivatives of AS4 (MAT $\alpha$ trp1 arg4 tyr7 ade6 ura3 MAL2) and AS13 (MATa leu2 ade6 ura3) (36). The strains with various his 4 mutations were constructed by the two-step transplacement procedure $(33,43)$. For the transformation step, plasmids phis4-519, phis4-17, phis4-712, and phis4-713 were treated with SpeI to target the integration (30). Subsequently, $\mathrm{Ura}^{-}$derivatives of the transformed strains were selected using 5-fluoro-orotate (3) and screened for those derivatives that were also $\mathrm{His}^{-}$. Table 1 summarizes the haploid strain constructions.

In the construction of yeast strains harboring two his4 mutant alleles, complementation tests (described below) were used to determine the genotype of $\mathrm{His}^{-}$transformants. The resulting haploid strains were analyzed by Southern blotting to ensure that the two-step transplacement procedure removed the vector sequences. The resulting haploids were then mated to generate diploids as shown in Table 2.

Media and genetic techniques. Standard procedures and media were used (35). Cells were sporulated on plates $(1 \%$

TABLE 2. Construction of related diploid strains

\begin{tabular}{lll}
\hline $\begin{array}{c}\text { Strain } \\
\text { name }\end{array}$ & \multicolumn{1}{c}{$\begin{array}{c}\text { Strain } \\
\text { construction }\end{array}$} & \multicolumn{1}{c}{ Relevant genotype $^{a}$} \\
\hline PD107 & AS13 $\times$ PD7 & HIS4/his4-519 \\
PD108 & AS13 $\times$ PD25 & HIS4/his4-712 \\
PD109 & AS13 $\times$ PD23 & HIS4/his4-713 \\
PD99 $b$ & PD98 $\times$ AS4 & his4-3133/HIS4 \\
DNY48 & DNY47 $\times$ AS4 & his4-IR9/HIS4 \\
PD93 & DNY47 $\times$ PD7 & his4-IR9/his4-519 \\
PD94 & DNY47 $\times$ PD23 & his4-IR9/his4-713 \\
PD95 & DNY47 $\times$ PD25 & his4-IR9/his4-712 \\
PD101 & PD100 $\times$ AS4 & his4-IR9 his4-3133/HIS4 \\
\hline
\end{tabular}

\footnotetext{
${ }^{a}$ Mutational changes within the HIS4 region.

b Strain constructed previously (6).
}

c Strain constructed previously (27). 
TABLE 3. Number of tetrads with aberrant segregations of his4 mutant alleles

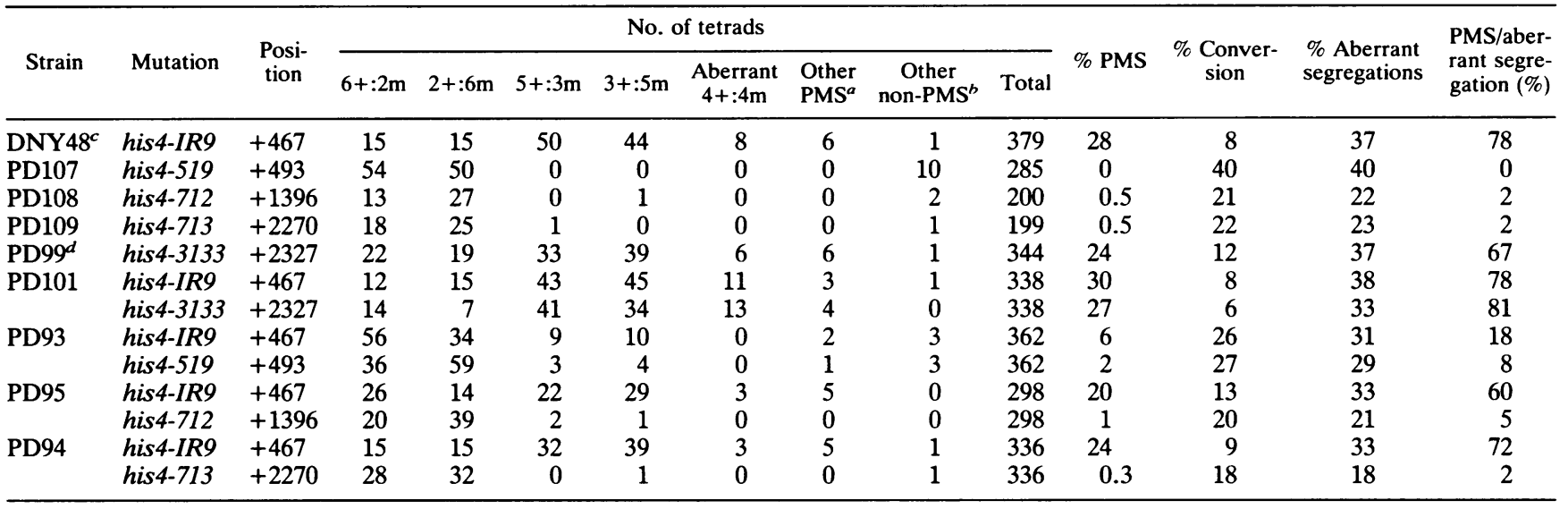

a Tetrads with more than one PMS event (other than an aberrant 4:4) or a conversion event and a PMS event.

$b$ Tetrads with two conversion events $(8+: 0 \mathrm{~m}$ or $0+: 8 \mathrm{~m})$.

c Reference 27.

d Data from reference 6.

potassium acetate, $0.1 \%$ yeast extract, $0.05 \%$ dextrose, $2 \%$ agar supplemented with adenine $[6 \mu \mathrm{g} / \mathrm{ml}])$ at $18^{\circ} \mathrm{C}(5)$. Tetrads were dissected on YPD plates (35) that were incubated at $32^{\circ} \mathrm{C}$. The resulting spore colonies were replica plated to various types of omission media.

For strains that were heterozygous for two his4 mutations (PD93, PD94, PD95, and PD101), complementation analysis was performed. This analysis is possible because the HIS4 gene has three distinct regions (HIS $4 A, H I S 4 B$, and $H I S 4 C)$, each of which codes for a separate enzymatic activity in a trifunctional protein (18). Since the his4-IR9 mutation is an in-frame insertion of a palindromic oligonucleotide in $H I S 4 A$, it is complemented by his4-712, his4-713, or his4280 , all of which represent his $4 C$ mutations. The his $4-\Delta 29$ mutation is an in-frame deletion which is his $4 A$, his $4 B$, HIS4C. It complements his4-712 and his4-713 but does not complement his4-IR9. The his4-519 mutation is a singlebase-pair insertion in $H I S 4 A$, resulting in a mutant phenotype for all three HIS4 enzymatic activities. Segregation of this allele was examined by using tester strains PD18 (MAT $\alpha$ SUF1 his4-Sal) and PD19 (MATa SUF1 his4-Sal); SUF1 is a dominant frameshift suppressor that suppresses his4-519 (34). The complementation and suppression tests were done by mating replicas of the original dissection plate to lawns of tester strains. The lawns were made by spraying a stationary-phase culture of a tester strain to YPD plates with a V\#1 Paasche airbrush. The plates were allowed to dry at $37^{\circ} \mathrm{C}$ for approximately $15 \mathrm{~min}$, and the replica of the spore colonies was imprinted on the lawn. After 12 to $16 \mathrm{~h}$ at $32^{\circ} \mathrm{C}$, the plate was replicated to a plate lacking histidine, and the replica was incubated overnight.

Statistical methods. Most of the statistical comparisons were done by using a contingency chi-square analysis; a chi-square value greater than 3.85 is significant at a level of $P<0.05$. In comparisons in which one or more classes had fewer than five events, we used the StatXact program (Cytel Software Corp.) to determine $\boldsymbol{P}$ values by the Fisher exact test.

\section{RESULTS}

Level and extent of the heteroduplex intermediate at HIS4. High-PMS alleles in yeast cells can be constructed by the insertion of palindromic oligonucleotides (28). When such insertions are heterozygous, about $70 \%$ of the aberrant segregation events are PMS events. In previous studies, we found that strains heterozygous for palindromic insertions near the 5' end of HIS4 have a PMS frequency of about $26 \%$ and a gene conversion frequency of about $8 \%$ (28). A strain with a palindromic insertion at the $3^{\prime}$ end of HIS4 (his4-3133) had similar frequencies of PMS $(26 \%)$ and gene conversion $(12 \%)(6)$. One interpretation of this result is that most of the heteroduplexes that include the $5^{\prime}$ region of HIS4 also include the $3^{\prime}$ region, a distance of $1.8 \mathrm{~kb}$. To test this possibility, we constructed a strain (PD101) that was heterozygous for two palindromic insertions, his4-3133 (at +2327) and his4-IR9 (at +467). The diploid was sporulated, and we examined the frequency of PMS and gene conversion events at both mutant sites (as described in Materials and Methods).

Of the 338 tetrads examined, 150 (44\%) had an aberrant segregation for at least one of the two his 4 mutations (Tables 3 and 4). The frequencies of aberrant segregation for the two mutant alleles were similar, $38 \%$ for his $4-I R 9$ and $33 \%$ for his 4-3133. These frequencies of aberrant segregation are similar to those observed for the same mutations in strains that were heterozygous for only one mutation (Table 3). Although there was a reduction in the level of gene conversion (from 12\% to 6\%) of his4-3133 in PD101 relative to PD99, the levels of PMS and conversion of his4-IR9 were similar in the presence (PD101) and absence (DNY48) of his4-3133. Thus, the two mutations in PD101 do not appear to interact in a way that grossly alters the pattern of recombination.

One common class of PMS tetrads (class 1; Fig. 1) was that in which both mutant alleles segregated $5+: 3 \mathrm{~m}(20$ tetrads) or both segregated $3+: 5 \mathrm{~m}$ (20 tetrads) (Table 4). In 35 of the 40 tetrads, the same spore colony was sectored (reflecting PMS) for both mutant alleles; this result strongly suggests that most of the class 1 tetrads represent a single heteroduplex that spans both alleles, rather than two independent heteroduplexes; one example of a class 1 tetrad is shown in Fig. 1. In PD101, one chromosome contains the wild-type HIS 4 allele and the other contains both mutant alleles. In all 35 tetrads in which a single spore colony sectored for both mutant alleles, one sector had the HIS4 
TABLE 4. Number of tetrads (and cosectoring patterns) in various classes of aberrant segregants for his4 mutant alleles in PD101

\begin{tabular}{|c|c|c|c|c|c|c|c|c|}
\hline & & & & hises & & & & \\
\hline & $5+: 3 m$ & $\underline{3+: 5 m}$ & $\underline{6+: 2 m}$ & $2+: 6 \mathrm{~m}$ & $\begin{array}{c}\text { Aberrant } \\
4+: 4 \mathrm{~m}\end{array}$ & Other $^{a}$ & $4+: 4 m$ & Totals \\
\hline $5+: 3 m$ & $20(18)^{b}$ & $6(3)$ & 0 & 2 & $5(5)$ & 1 & 7 & 41 \\
\hline $3+: 5 m$ & $2(1)$ & $20(17)$ & 0 & 4 & 0 & $1(1)$ & 7 & 34 \\
\hline $6+: 2 m$ & 2 & 1 & 7 & 0 & 0 & 0 & 4 & 14 \\
\hline $2+: 6 m$ & 0 & 0 & 0 & 4 & 0 & 2 & 1 & 7 \\
\hline $\begin{array}{c}\text { Aberrant } \\
4+: 4 m\end{array}$ & 0 & $6(4)$ & 0 & 1 & $5(9)$ & 0 & 1 & 13 \\
\hline Other $^{b}$ & $3(2)$ & 0 & 0 & 0 & $1(2)$ & 0 & 0 & 4 \\
\hline $4+: 4 m$ & 16 & 12 & 5 & 4 & 0 & 0 & $188^{c}$ & 225 \\
\hline Totals & 43 & 45 & 12 & 15 & 11 & 4 & 208 & 338 \\
\hline
\end{tabular}

${ }^{a}$ Includes $8+: 0 \mathrm{~m}, 0+: 8 \mathrm{~m}$, ab6 $+: 2 \mathrm{~m}$, ab2 $+: 6 \mathrm{~m}, 7+: 1 \mathrm{~m}$, and $1+: 7 \mathrm{~m}$ tetrads and tetrads with three PMS events for one or more alleles.

${ }^{b}$ Each number without parentheses is the number of tetrads in each class. The number in parentheses indicates the number of spore colonies among these tetrads that were sectored in the same direction for both his $4-I R 9$ and his4-3133. For example, there were six tetrads that segregated 5+:3m for his $4-3133$ and $3+: 5 \mathrm{~m}$ for his 4-IR9. In three of six tetrads, the same spore colony sectored for both his4-IR9 and his4-3133.

$c$ There were five crossovers between the his 4 mutations in this class.

allele and the other had both mutant alleles. This result also supports the conclusion that these tetrads represent transfer of a single continuous strand of DNA from one chromosome to the other, without correction of either of the resulting two mismatches.

Another common class of aberrant segregation pattern (class 2; examples shown in Fig. 1) was that in which one mutant allele showed PMS (either $5+: 3 \mathrm{~m}$ or $3+: 5 \mathrm{~m}$ ) and the other allele showed normal Mendelian segregation $(4+: 4 \mathrm{~m})$. In 28 tetrads, the his $4-I R 9$ allele had a single PMS event (16, $5+: 3 \mathrm{~m} ; 12,3+: 5 \mathrm{~m}$ tetrads), with the his 4-3133 allele segregating $4+: 4 \mathrm{~m}$. In 14 tetrads, the his $4-3133$ allele had a single PMS event $(7,5+: 3 \mathrm{~m} ; 7,3+: 5 \mathrm{~m}$ tetrads), with the his4-IR 9 allele segregating $4+: 4 \mathrm{~m}$. Such tetrads could reflect heteroduplexes that involved only one of the two alleles and failure to repair the single mismatch (class $2 \mathrm{a}$; Fig. 1). Alternatively, these tetrads could reflect heteroduplexes that span both alleles in which one of the resulting mismatches is repaired to restore $4+: 4 \mathrm{~m}$ segregation and the other mismatch is unrepaired (class 2b; Fig. 1).

A third common class (class 3 ) were tetrads in which both mutant alleles segregated $6+: 2 \mathrm{~m}$ (seven tetrads) or both segregated $2+: 6 \mathrm{~m}$ (four tetrads). Such tetrads could reflect mismatch repair events in a single heteroduplex spanning both alleles (Fig. 1). Alternatively, this class could reflect a conversion event that did not involve a heteroduplex intermediate (for example, gene conversion as the result of repair of a double-stranded gap [38]).

The three classes described above can be explained by formation of single asymmetric heteroduplexes in which the resulting mismatches are either repaired or left unrepaired. Most of the other classes of aberrant segregation involve more than one sector per tetrad and, therefore, presumably more than one heteroduplex. The frequency of these events is approximately that expected if the heteroduplexes are initiated independently. More specifically, if the frequency of tetrads with single PMS events at the his4-IR9 allele is $26 \%$ (88 of 338), the expected frequency of tetrads with two PMS events at that site is about $0.26^{2}$, or $7 \%$. The expected number of tetrads with two PMS events at that site is, therefore, about $23(0.07 \times 338)$. We observed 14 such tetrads (Table 3 ).

The most common class of tetrads with two PMS events (class 4) were those in which one allele had an aberrant $4: 4$ segregation $\left(1 \mathrm{His}^{+}\right.$spore colony: 2 sectored $\mathrm{His}^{+} / \mathrm{His}^{-}$ colonies: $1 \mathrm{His}^{-}$colony) and the other segregated $5+: 3 \mathrm{~m}$ or $3+: 5 \mathrm{~m}$, or tetrads in which both alleles had the aberrant $4: 4$ segregation pattern (Table 4 ). The first of these classes could 


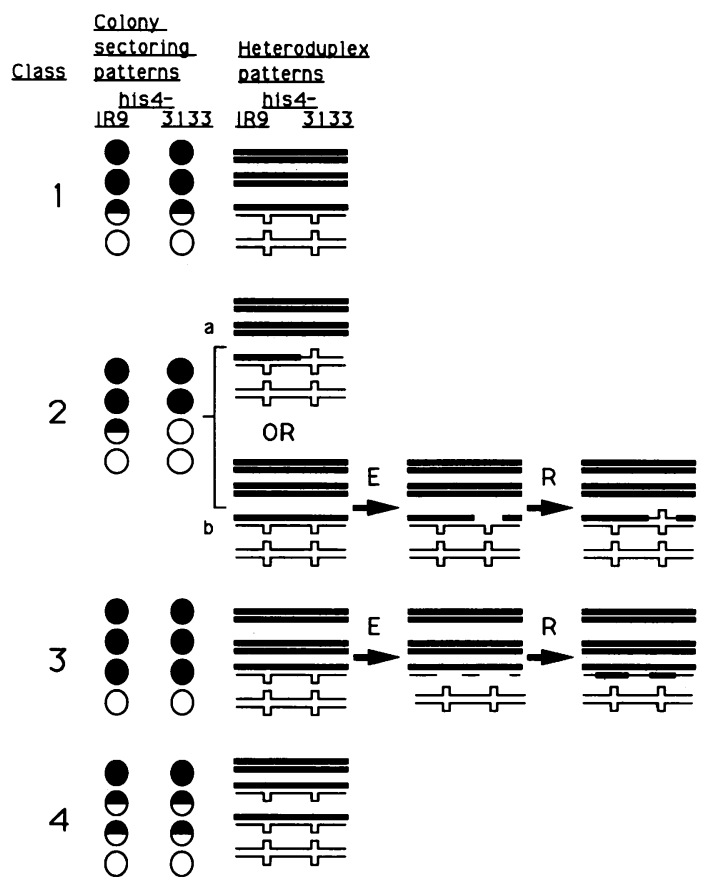

FIG. 1. Examples of colony sectoring patterns and interpretive heteroduplex patterns for various classes of aberrant segregants at the HIS4 locus in the diploid strain PD101. In PD101, one chromosome contained a wild-type HIS4 gene and the other contained two palindromic insertions (his4-IR9 near the $5^{\prime}$ end of the gene and his4-3133 near the $3^{\prime}$ end of the gene). The tetrads were dissected, and spores were allowed to form colonies on plates containing a rich growth medium. The resulting colonies were mated to various tester strains and replica plated to medium lacking histidine (as described in Materials and Methods) in order to determine the segregation pattern of each mutant allele separately. Black circles indicate $\mathrm{His}^{+}$ colonies, and white circles indicate $\mathrm{His}^{-}$colonies. Sectored circles reflect PMS events. Thus, in the example of a class 1 aberrant segregation shown (classes are defined in the text), for both alleles, two of the spore colonies have only the wild-type allele and one has only the mutant allele. In this example, the same spore colony sectors for both mutant alleles. The simplest interpretation of this sectoring pattern is that one of the wild-type HIS4 genes donated a single DNA strand to a mutant gene; the resulting heteroduplex spans both mutant alleles. In the heteroduplex diagrams, the wildtype and mutant DNA strands are indicated by thick and thin lines, respectively. The mutant substitutions are drawn as hairpins, since palindromic insertions would be expected to form such structures in heteroduplexes (28). The class 2 sectored colonies could be formed in two different ways: a heteroduplex covering only one allele and failure to repair the resulting mismatch (class $2 \mathrm{a}$ ), or a heteroduplex covering both mutations, followed by correction of only one of the mismatches (class $2 b$ ). Step $E$ represents the excision of the mismatch, and step R shows repair of the resulting gap. Class 3 and class 4 tetrads represent gene conversion events and aberrant $4: 4$ segregation events, respectively.

reflect formation of two heteroduplexes spanning both mutant alleles, followed by correction of one of the mismatches. Alternatively, this class could reflect independent formation of two heteroduplexes, one involving only one of the mutant alleles and one spanning both. There is an interesting asymmetry in this class; all tetrads with a single PMS event at his4-IR9 and two PMS events at his4-3133 represented $3+: 5 \mathrm{~m}$ tetrads at his4-IR9, whereas the comparable class of tetrads with a single event at his $4-3133$ had only $5+: 3 \mathrm{~m}$ tetrads. One possible explanation for this finding is that mismatches involving the his4-IR9 mismatch are more frequently repaired to generate a mutant gene than are mismatches involving the his4-3133 hairpin. A slight bias of this sort is seen in the ratios of 6:2/2:6 tetrads in PD101, 12/15 for his4-IR9, and 14/7 for his4-3133 (Table 3).

The class of tetrads in which both alleles showed the aberrant 4:4 segregation pattern is likely to represent independent formation of two heteroduplexes, each spanning both mutations (Fig. 1). In 14 of the 16 class 4 tetrads, the sectoring patterns were consistent with the formation of one or more heteroduplexes that span both mutant alleles (Table 4).

In summary, about half of the spore colonies that sectored for the his4-IR9 allele cosectored at his4-3133; similarly, about half of the spore colonies that sectored for the his43133 allele cosectored at his4-IR9. These results indicate that approximately half of the heteroduplexes initiated at the HIS4 locus span both mismatches, a distance of $1.8 \mathrm{~kb}$. This estimate is minimal because some tetrads in classes 2 and 3 may also represent heteroduplexes that span both mutant alleles.

Length of the meiotic excision repair tract. As discussed in the introduction, in a number of systems, it has been shown that the repair of a mismatch involves a local excision of DNA flanking the mismatch. One method of determining the size of this excision tract is to examine the effect of mutant alleles that lead to well-repaired mismatches (low-PMS alleles) on the segregation of closely linked alleles that lead to poorly repaired mismatches (high-PMS alleles). If the excision tract initiated from the low-PMS allele includes the high-PMS allele, then the level of PMS for the high-PMS allele should be reduced. The rationale for this type of analysis is shown in Fig. 2.

As shown in Table 3, a strain that is heterozygous for the high-PMS allele his4-IR9 (at +467 ) by itself shows $28 \%$ PMS and $8 \%$ gene conversion per unselected tetrad (27). We made three strains heterozygous for his4-IR9 and one of three mutant alleles that contain the insertion of a single $G$ at different positions in the gene (his4-519 [at +493], his4-712 [at +1396$]$, and his4-713 [at +2270$])$. As shown in Table 3, these single-base insertions (when alone) show little or no PMS (less than $2 \%$ of the aberrant segregations), indicating that they lead to well-repaired mismatches in meiotic heteroduplexes. The aberrant segregation patterns of his4-IR 9 in the presence of each of these alleles are shown in Tables 3 , 5,6 , and 7 . It should be noted that, unlike the PD101 diploid, in the three diploids used in this analysis, one chromosome had the his4-IR9 mutation and the other had the low-PMS his4 mutation.

The his 4-519 mutation, located 26 bp from his4-IR9, reduces the PMS per tetrad of his4-IR9 about fivefold (from $28 \%$ to $\left.6 \%\left[x^{2}=65\right]\right)$. This decrease is compensated for by an increase in the rate of gene conversion of his4-IR 9 from $8 \%$ to $26 \%$ of the total tetrads $\left(\chi^{2}=40\right)$. Most of his4-IR9 gene conversions (97\% of the $6+: 2 \mathrm{~m}$ and $2+: 6 \mathrm{~m}$ segregations) were coconversions with his4-519 (Table 5). The coconversions $(6+: 2 \mathrm{~m}$ and $2+: 6 \mathrm{~m}$ events only) between his4-IR9 and his4-519 represented $24 \%$ of the total tetrads. The dramatic reduction in PMS for his4-IR9 and the concomitant increase in gene conversion represent the effects expected if the excision tract initiated at the well-repaired his4-519 mismatch almost always includes the his4-IR9 mismatch.

The allele his4-712, located 929 bp from his4-IR9, decreased the PMS of his4-IR9 from $28 \%$ to $20 \%$ of the total tetrads $\left(\chi^{2}=6.3\right)$ and increased the conversion rate of 
a.

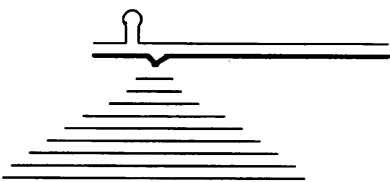

b.

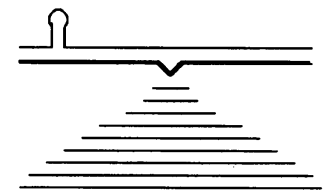

C.

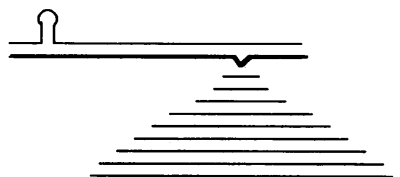

FIG. 2. Effects of a well-repaired mismatch on neighboring poorly repaired mismatches. Shown are heteroduplexes formed between two mutant his4 genes, one containing the his4-IR9 mutation (resulting in a poorly repaired mismatch) and one containing a well-repaired mismatch. The positions of the mismatches are indicated by the hairpin (his4-IR9) or triangles (other mismatches). The horizontal lines emanating from each well-repaired mismatch represent regions of DNA that are excised during excision of the mismatch. We show these excision tracts as occurring bidirectionally from the mismatch, although there are no data supporting this pattern. In addition, the distribution of tract lengths is not known. If the distribution of tract lengths is the same from each mismatch, then one would expect that an excision repair tract initiating from the well-repaired mismatch would affect the segregation of the poorly repaired mismatch in a distance-dependent manner. The figure is drawn to reflect the approximate distances between his4IR 9 and his4-519 (a), his4-IR9 and his4-712 (b), and his4-IR9 and his4-713 (c).

his 4-IR9 from $8 \%$ to $13 \%$ of the total tetrads $\left(\chi^{2}=4.3\right)$. Most of his4-IR9 gene conversions $(63 \%$ of the $6+: 2 \mathrm{~m}$ and $2+: 6 \mathrm{~m}$ segregations) were coconversions with his4-712 (Table 6). The coconversions $(6+: 2 \mathrm{~m}$ and $2+: 6 \mathrm{~m}$ events only) between his4-IR9 and his4-712 represented $8 \%$ of the total tetrads. Since the PMS level of his4-IR9 was lowered by about one-third by the presence of his4-712, we suggest that approximately one-third of the excision tracts initiated at his4-712 include his4-IR9. This estimate is a minimal one, since not all of the heteroduplexes that include the his4-IR9 mismatch will also include his4-712.

The allele his4-713, located 1,803 bp from his4-IR9, had no significant effect on the segregation of his4-IR9 (Table 7). In the presence of his4-713, PMS per tetrad for his4-IR9 remained high at $24 \%$ (a rate statistically not different from $28 \%$ PMS per tetrad for his4-IR9 alone $\left.\left[\chi^{2}=2.0\right]\right)$. Although his 4-713 also had no significant effect on gene conversion of his4-IR9 $\left(\chi^{2}=0.13\right)$, the two alleles still showed some coconversion $(43 \%$ of all the $6+: 2 \mathrm{~m}$ and $2+: 6 \mathrm{~m}$ events at his $4-I R 9$ were coconversions with his4-713). The coconversions $(6+: 2 \mathrm{~m}$ and $2+: 6 \mathrm{~m}$ events only) between his4-IR9 and his $4-713$ represented $3.9 \%$ of the total tetrads. These results indicate that excision tracts initiated at his4-713 usually do not include his4-IR9.

Induction of PMS at a well-repaired mismatch by a closely linked poorly repaired mismatch. Although previous studies $(12,15,23)$ showed that a well-repaired allele can reduce the PMS of a nearby poorly repaired allele, the poorly repaired allele was not observed to increase the frequency of PMS for the well-repaired alleles. In contrast, we found that his4-IR 9 significantly increased the PMS rate of his4-519 (located 26 bp away) from $0 \%$ to $2 \%$ of total tetrads (Fisher exact test; $P$ $=0.009$ ). As shown in Table 5, each of the PMS events at his4-519 had a co-PMS event at his4-IR9. No statistically significant increase in PMS was measured in the presence of his4-IR9 for the two alleles his4-712 (929 bp downstream of his4-IR9) and his4-713 (1,803 bp downstream of his4-IR9) (Fisher exact test; $P=0.34$ and 0.47 , respectively).

\section{DISCUSSION}

From our meiotic analysis of mutant his 4 alleles separated by various known physical distances, we conclude that (i) meiotic heteroduplexes often span most of the HIS4 gene (a distance of $1.8 \mathrm{~kb}$ ), (ii) excision-repair tracts almost always extend $26 \mathrm{bp}$ and about one-third of the time extend $900 \mathrm{bp}$, and (iii) certain high-PMS alleles can elevate the frequency of PMS for very closely linked low-PMS alleles. Each of these conclusions will be discussed further below.

As shown in Table 4, approximately half of the PMS events at his4-IR9 were associated with a PMS event at his4-3133. The simplest interpretation of this observation is that the co-PMS pattern reflects a continuous asymmetric heteroduplex spanning the $1.8-\mathrm{kb}$ distance between the mutant alleles. As indicated in Fig. 1, those tetrads with PMS events involving only one of the two mutant alleles could reflect either heteroduplexes that cover only one allele or repair of one of the two mismatches. The observation that the level of PMS was similar for his4-IR9 at the 5' end of the gene and his4-3133 at the $3^{\prime}$ end of the gene is consistent with previous conclusions based on the analysis of strains containing single his 4 mutations (6). It should be emphasized that the high level of PMS observed for his4-3133 does not reflect the ability of palindromic mutations to initiate heteroduplexes. The levels of aberrant segregation of his4-IR 9 are identical in the presence and absence of his4-3133. In addition, the level of aberrant segregation for his4-3133 is identical to that observed for the high-PMS his4-200 allele, a base substitution at the $3^{\prime}$ end of HIS4 (6).

The well-repaired alleles his4-519, his4-712, and his4-713 are located within this $1.8-\mathrm{kb}$ region known to be frequently spanned by meiotic heteroduplexes. Thus, when his4-IR9 is involved in a meiotic heteroduplex, this heteroduplex should span the site of each of these alleles more than half of the time. We found that the presence of the heterozygous his4-519 allele $($ at +493$)$ greatly decreased the PMS and increased the gene conversion rate of his4-IR9 (at +467). This observation suggests that the repair of his 4-519 mismatches in a heteroduplex stimulates the repair of his4-IR 9 mismatches in the same heteroduplex. Since the reduction in the frequency of PMS for his4-IR9 (from $28 \%$ to $6 \%$ of the total tetrads) is nearly balanced by the increase in gene conversion (from $8 \%$ to $26 \%$ ), we conclude the most of the repair events induced by his4-519 represent conversion-type repair rather than restoration-type repair. This conclusion supports previous studies indicating that most repair events near the 5' end of HIS4 represent conversion-type repair (5). In addition, these results indicate that (i) mismatches resulting from heterozygous palindromic insertions are not well recognized by the meiotic mismatch repair system and (ii) 
TABLE 5. Number of tetrads (and cosectoring patterns) in various classes of aberrant segregants for his 4 mutant alleles in PD93 ${ }^{a}$

\begin{tabular}{|c|c|c|c|c|c|c|c|c|}
\hline & & & $\frac{\text { his4-IR9 }}{T_{+467}}$ & & & & & \\
\hline & $\underline{5+: 3 m}$ & $3+5 \mathrm{~m}$ & $6+: 2 \mathrm{~m}$ & $2+: 6 \mathrm{~m}$ & $\begin{array}{c}\text { Aberrant } \\
4+: 4 m\end{array}$ & Other & $4+: 4 \mathrm{~m}$ & Totals \\
\hline $5+: 3 \mathrm{~m}$ & 0 & $3(3)$ & 0 & 0 & 0 & 0 & 0 & 3 \\
\hline $3+: 5 m$ & $4(4)$ & 0 & 0 & 0 & 0 & 0 & 0 & 4 \\
\hline $2+: 6 \mathrm{~m}$ & 2 & 0 & 56 & 0 & 0 & 0 & 1 & 59 \\
\hline $\begin{array}{c}\text { Aberrant } \\
4+: 4 m\end{array}$ & 0 & 0 & 0 & 0 & 0 & 0 & 0 & 0 \\
\hline Other & 0 & 0 & 0 & 0 & 0 & $4(1)$ & 0 & 4 \\
\hline $4+: 4 m$ & 3 & 3 & 0 & 3 & 0 & 0 & $247^{b}$ & 256 \\
\hline Totals & 9 & 10 & 56 & 34 & 0 & 5 & 248 & 362 \\
\hline
\end{tabular}

${ }^{a}$ See Table 4, footnotes $a$ and $b$.

$b$ There were no crossovers between the his 4 mutant alleles in this class.

such mismatches can be repaired by excision tracts initiated elsewhere.

In the presence of his4-712, the frequency of PMS of his4-IR9 drops by one-third, with a comparable elevation in gene conversion, indicating that repair tracts involved in the repair of mismatches at his 4-712 span the 929 bp between the two alleles about one-third of the time. This result also shows that mismatches are not repaired in the same way in every tetrad. No effect of the his4-713 mutation on the PMS frequency of his4-IR 9 was observed, indicating that excision repair tracts usually do not span a distance of $1.8 \mathrm{~kb}$. It should be pointed out, however, that not all of the heteroduplexes that involve his4-IR9 also include the $3^{\prime}$ end of the gene; as discussed above, we estimate that at least half of the heteroduplexes span the $5^{\prime}$ and $3^{\prime}$ alleles. PMS events at his4-IR9 that do not involve a heteroduplex at his4-713 would not be expected to be affected by the his 4-713 mutation. Consequently, we cannot exclude the possibility that some fraction (one-third or less) of the excision repair events initiated at the his4-713 mismatch extend to his4-IR9.

The frequency of repair tracts reaching his4-IR9 might be influenced by the nature of sequences or chromosomal structures between his4-713 and his4-IR9, as well as by the physical distance between the alleles. A sequence that inhibits meiotic mismatch repair has been reported (40), although this sequence is not present in the HIS4 gene. Although it is not possible to calculate accurately an average tract length on the basis of our data (since we do not accurately know the distribution of tract lengths and we do not know whether the excision events occur unidirectionally or bidirectionally from the mismatch), if we assume a normal distribution of tract lengths, we estimate that the average excision repair tract length is propagated 500 to $800 \mathrm{bp}$ from the initiating mismatch (either uni- or bidirectionally).

Two additional caveats should be mentioned. First, it is possible that the mismatch signals the excision event, but the excision tract does not initiate at the mismatch. Second, the plasmid-borne HIS 4 gene used in our study to introduce mutations was not derived from our yeast strain; therefore, additional hidden heterozygous markers may be present in our strains. Although this possibility cannot be excluded, it is unlikely for several reasons. First, such hidden heterozygous markers would be expected to obscure the distancedependent effects of his 4-519, his4-712, and his4-713 on the PMS frequency of his4-IR9. Second, the levels of PMS for both palindromic insertions in the strain PD101 were both very high, about $80 \%$ of the total aberrant segregants; hidden heterozygosity should lower the frequency of PMS relative to gene conversion.

Our results agree with those of Fogel et al. $(12,13)$, indicating that the meiotic mismatch repair tracts in $S$. cerevisiae are frequently more than $214 \mathrm{bp}$ in length; this agreement suggests that the unusually high rate of aberrant segregation of HIS4 seen in our genetic background does not 
TABLE 6. Number of tetrads (and cosectoring patterns) in various classes of aberrant segregants for his 4 mutant alleles in PD95 ${ }^{a}$

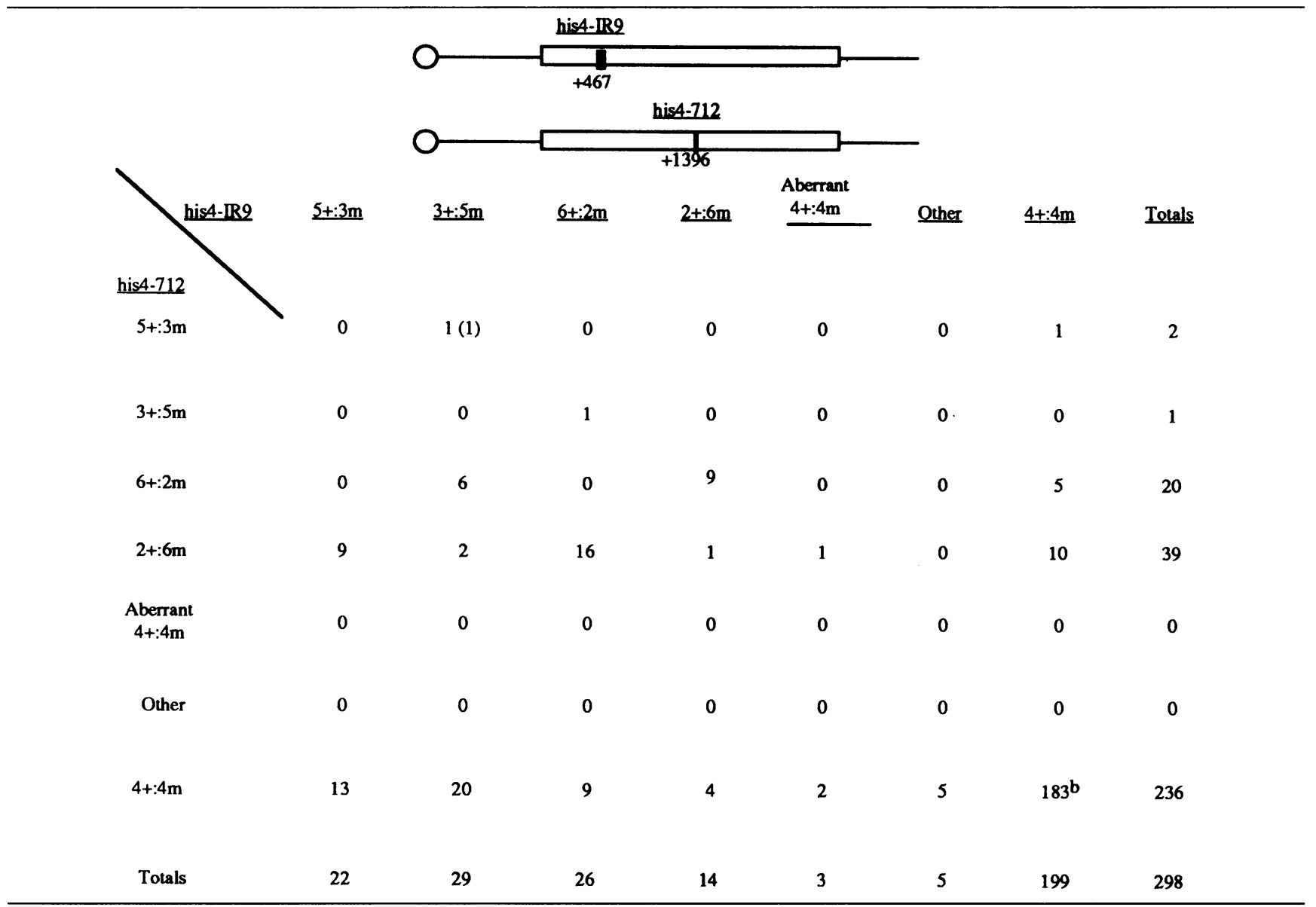

${ }^{a}$ See Table 4, footnotes $a$ and $b$.

$b$ There were six crossovers in this class between the his 4 mutant alleles.

reflect any gross alteration in the length of excision repair tracts. The results also agree with the mitotic study of Bishop and Kolodner (2) which indicates that mismatches separated by $0.9 \mathrm{~kb}$ are sometimes not involved in the same repair tract during mitotic repair. Since the meiotic and mitotic repair systems in $S$. cerevisiae share the same specificity toward individual base mismatches $(2,5,21)$, these two systems are likely to be very similar or identical. Meiotic repair tracts in $S$. cerevisiae are similar in length to those in $E$. coli, which have an average length of $3 \mathbf{~ k b ~ ( 3 9 ) , ~}$ and are shorter than those in $S$. pneumoniae, which range from 5 to $10 \mathrm{~kb}(14,24)$.

As discussed previously, if all gene conversion events reflect mismatch repair in heteroduplex DNA, the length and continuity of conversion tracts will be a function of the lengths of the heteroduplex and the excision repair tract. In $S$. cerevisiae, the average meiotic gene conversion tract length has been estimated to be $1.5 \mathrm{~kb}$ at the $M A T$ locus (4), $2.3 \mathrm{~kb}$ at $U R A 3(19)$, and $3.7 \mathrm{~kb}$ in the LEU2-CEN3 region (37). In one study (37), $20 \%$ of all conversion tracts measured were more than $5 \mathrm{~kb}$. In all these studies, the conversion tracts were almost always continuous (sites showing gene conversion were not separated by sites showing normal Mendelian segregation). Although our studies indicate that the length of the heteroduplexes may be sufficient to account for most of the conversion data, it is not clear that our data explain the continuity of very long (greater than $5 \mathrm{~kb}$ ) conversion tracts. Possible explanations of this discrepancy are as follows: (i) long meiotic conversion tracts might reflect some mechanism other than heteroduplex formation and repair (such as the repair of double-stranded gaps with the sequences from a homologous chromosome [38]), (ii) one strand of a heteroduplex may be tagged for excision by a mechanism analogous to methyl-directed repair in $E$. coli, and (iii) the lengths of repair tracts are different in different regions of the genome.

Our experiments showed that the PMS rate of his4-IR9 was severely reduced by the presence of his4-519 (an allele that rarely shows PMS). In addition, the PMS rate of his4-519 is increased by the presence of his4-IR9. The effects these alleles have on each other are not of equal magnitude. The decrease in PMS of his4-IR9 involves about $20 \%$ of the total tetrads, whereas the increase in PMS of his4-519 involves only $2 \%$ of the tetrads. Although decreases in PMS by the presence of another allele have been seen previously $(12,15,23)$, the induction of PMS of one allele by the presence of another allele has not yet been reported.

One of the differences between this study and previous studies is that his4-IR9 is a palindromic insertion. Evidence indicates that palindromic insertions show high PMS as a result of their ability to undergo intrastrand base pairing resulting in a hairpin configuration (28). Although many of 
TABLE 7. Number of tetrads (and cosectoring patterns) in various classes of aberrant segregants for his4 mutant alleles in PD94 ${ }^{a}$

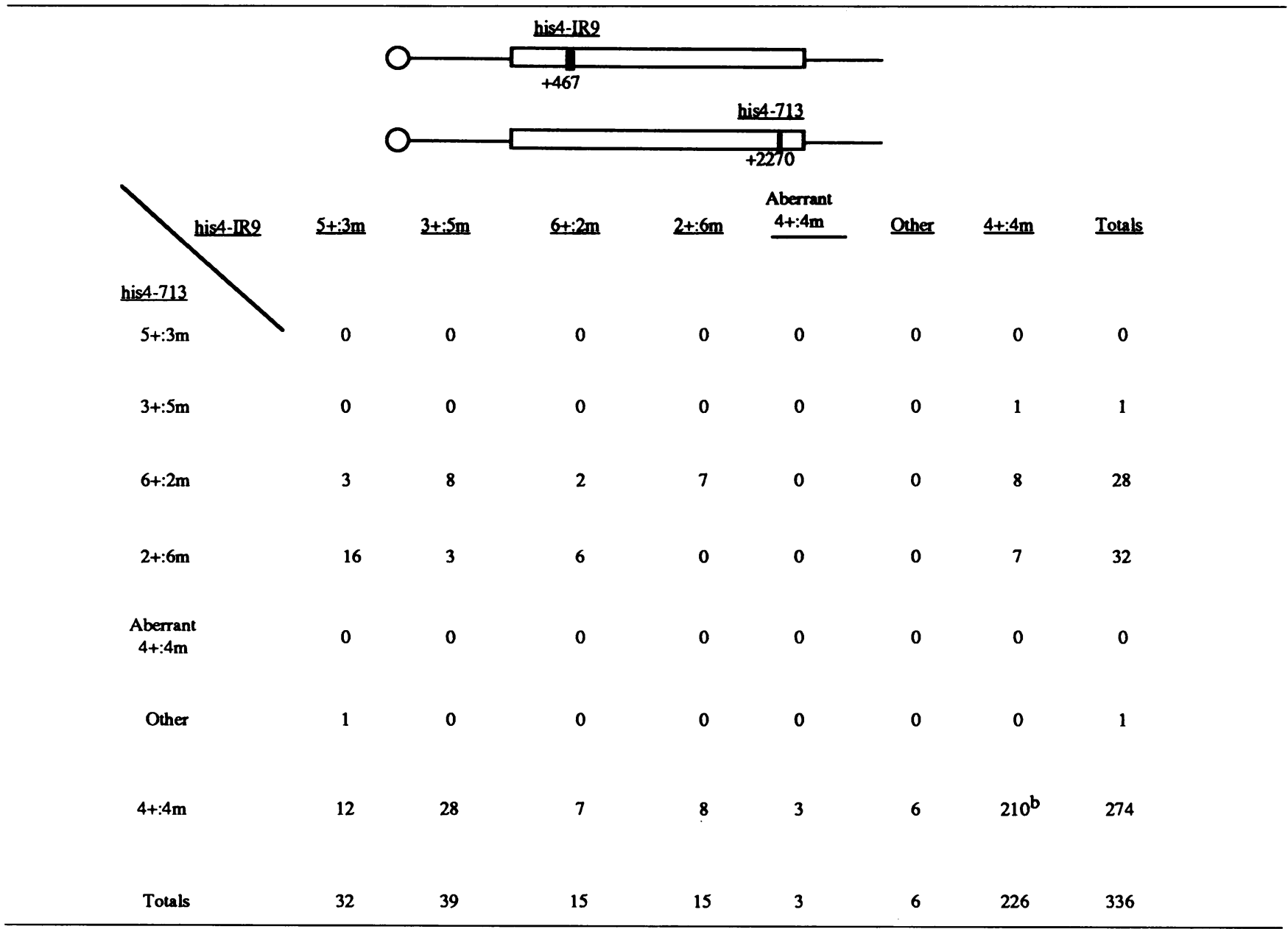

a See Table 4, footnotes $a$ and $b$.

$b$ There were three crossovers between the his 4 mutant alleles in this class.

the his4-IR9 hairpins are repaired in the presence of his4$519,38 \%$ of the hairpins which escape repair are involved with a co-PMS event at his4-519. All of the PMS events at his4-519 are co-PMS events with his4-IR9. The distance separating these alleles is $26 \mathrm{bp}$; no induction of PMS was seen for alleles at a further distance. One interpretation of this result is that there are proteins bound to the hairpin that inhibit its repair or recognition and these proteins can sometimes inhibit the repair of very closely linked mutations. Since previous studies did not investigate mutant alleles that were extremely closely linked, it is also possible that the reciprocal effects that we have detected are due to the short distance between the mismatches rather than the type of high-PMS allele.

\section{ACKNOWLEDGMENTS}

This research was supported by NIH grant GM24110 and an NIH training grant at the University of Chicago (P.D.).

We thank D. K. Nag for providing strains for the study and relevant unpublished data. We thank all members of the Petes laboratory (particularly M. White) for useful discussions and comments on the manuscript.

\section{REFERENCES}

1. Arndt, K. T., C. Styles, and G. R. Fink. 1987. Multiple global regulators control HIS4 transcription in yeast. Science 237:874 880 .

2. Bishop, D. K., and R. D. Kolodner. 1986. Repair of heteroduplex plasmid DNA after transformation into Saccharomyces cerevisiae. Mol. Cell. Biol. 6:3401-3409.

3. Boeke, J. D., F. Lacroute, and G. R. Fink. 1984. A positive selection for mutants lacking orotidine-5'-phosphate decarboxylase activity in yeast: 5-fluoro-orotic acid resistance. Mol. Gen. Genet. 197:345-346.

4. Borts, R. H., and J. E. Haber. 1989. Length and distribution of meiotic gene conversion tracts and crossovers in Saccharomy. ces cerevisiae. Genetics 123:69-80.

5. Detlofi, P. D., J. Sieber, and T. D. Petes. 1991. Repair of specific base pair mismatches formed during meiotic recombination in the yeast Saccharomyces cerevisiae. Mol. Cell. Biol. 11:737745.

6. Detloff, P. D., M. A. White, and T. D. Petes. Unpublished data.

7. DiCaprio, L., and P. J. Hastings. 1976. Gene conversion and intragenic recombination at the SUP6 locus and the surrounding region in Saccharomyces cerevisiae. Genetics 84:697-721.

8. Donahue, T. F., and A. M. Cigan. 1988. Genetic selection for mutations that reduce or abolish ribosomal recognition of the HIS4 translational initiator region. Mol. Cell. Biol. 8:2955-2963. 
9. Donahue, T. F., P. J. Farabaugh, and G. R. Fink. 1982. The nucleotide sequence of the HIS4 region of yeast. Gene 18:47-59.

10. Esposito, M. S. 1971. Post-meiotic segregation in Saccharomyces cerevisiae. Mol. Gen. Genet. 111:297-299.

11. Fogel, S., and R. K. Mortimer. 1969. Informational transfer in meiotic gene conversion. Proc. Natl. Acad. Sci. USA 62:96103.

12. Fogel, S., R. K. Mortimer, and K. Lusnak. 1981. Mechanisms of meiotic gene conversion, or "wanderings on a foreign strand," p. 289-339. In J. Strathern, E. W. Jones, and J. Broach (ed.), The molecular biology of the yeast Saccharomyces, vol. 1. Cold Spring Harbor Laboratory, Cold Spring Harbor, N.Y.

13. Fogel, S., R. K. Mortimer, K. Lusnak, and F. Tavares. 1979. Meiotic gene conversion-a signal of the basic recombination events in yeast. Cold Spring Harbor Symp. Quant. Biol. 43: 1325-1341.

14. Guild, W. R., and N. B. Shoemaker. 1976. Mismatch correction in pneumococcal transformation: donor length and hex-dependent marker efficiency. J. Bacteriol. 125:125-135.

15. Hastings, P. J. 1984 . Measurement of restoration and conversion: its meaning for the mismatch repair hypothesis of conversion. Cold Spring Harbor Symp. Quant. Biol. 49:49-53.

16. Hastings, P. J. 1988. Conversion events in fungi, p. 397-428. In R. Kucherlapati and G. Smith (ed.), Meiosis. American Society for Microbiology, Washington, D.C.

17. Holliday, R. 1964. A mechanism for gene conversion in fungi. Genet. Res. 5:282-304.

18. Jones, E. W., and G. R. Fink. 1981. Regulation of amino acids and nucleotide biosynthesis in yeast, p. 181-299. In J. N. Strathern, E. W. Jones, and J. R. Broach (ed.), The molecular biology of the yeast Saccharomyces: metabolism and gene expression. Cold Spring Harbor Laboratory, Cold Spring Harbor, N.Y.

19. Judd, S. R., and T. D. Petes. 1988. Physical lengths of meiotic and mitotic gene conversion tracts in Saccharomyces cerevisiae. Genetics 118:401-410.

20. Kraft, R., J. Tardif, K. S. Krauter, and L. A. Leinwand. 1988. Using mini-prep plasmid DNA for sequencing double-stranded templates with Sequenase. Bio/Technology 6:544-545.

21. Kramer, B., W. Kramer, M. S. Williamson, and S. Fogel. 1989. Heteroduplex DNA correction in Saccharomyces cerevisiae is mismatch specific and requires functional PMS genes. Mol. Cell. Biol. 9:4432-4440.

22. Kurnit, D. M. 1989. Escherichia coli recA deletion strains that are highly competent for transformation and for in vivo phage packaging. Gene 82:313-315.

23. Leblon, G., and J.-L. Rossignol. 1973. Mechanism of gene conversion in Ascobolus immersus. III. The interactions of heteroalleles in the conversion process. Mol. Gen. Genet. 122:165-182.

24. Mejean, V., and J. P. Claverys. 1984. Effect of mismatched base pairs on the fate of donor DNA in transformation of Streptococcus pneumoniae. Mol. Gen. Genet. 197:467-471.

25. Meselson, M., and C. Radding. 1975. A general model for genetic recombination. Proc. Natl. Acad. Sci. USA 72:358-361.

26. Modrich, P. 1987. DNA mismatch correction. Annu. Rev. Biochem. 56:435-466.

27. Nag, D. K., and T. D. Petes. 1991. Seven base-pair inverted repeats in DNA form stable hairpins in vivo in S. cerevisiae. Genetics 129:669-673.

28. Nag, D. K., M. A. White, and T. D. Petes. 1989. Palindromic sequences in heteroduplex DNA inhibit mismatch repair in yeast. Nature (London) 340:318-320.

29. Nagawa, F., and G. R. Fink. 1985. The relationship between the "TATA" sequence and transcription initiation sites at the HIS4 gene of Saccharomyces cerevisiae. Proc. Natl. Acad. Sci. USA 82:8557-8561.

30. Orr-Weaver, T. L., J. W. Szostak, and R. Rothstein. 1981. Yeast transformation: a model system for the study of recombination. Proc. Natl. Acad. Sci. USA 78:6354-6358.

31. Radding, C. M. 1982. Homologous pairing and strand exchange in genetic recombination. Annu. Rev. Genet. 16:405-437.

32. Rossignol, J.-L., A. Nicolas, H. Hamza, and A. Kalogeropoulos. 1988. Recombination and gene conversion in Ascobolus, p. 23-72. In K. B. Low (ed.), The recombination of genetic material. Academic Press, New York.

33. Scherer, S., and R. W. Davis. 1979. Replacement of chromosome segments with altered DNA sequences constructed in vitro. Proc. Natl. Acad. Sci. USA 76:4951-4955.

34. Sherman, F. 1981. Suppression in the yeast Saccharomyces cerevisiae, p. 463-486. In J. N. Strathern, E. W. Jones, and J. R. Broach (ed.), The molecular biology of the yeast Saccharomyces: metabolism and gene expression. Cold Spring Harbor Laboratory, Cold Spring Harbor, N.Y.

35. Sherman, F., G. R. Fink, and J. B. Hicks. 1982. Methods in yeast genetics. Cold Spring Harbor Laboratory, Cold Spring Harbor, N.Y.

36. Stapleton, A., and T. D. Petes. 1991. The Tn3 $\beta$-lactamase gene acts as a hotspot for meiotic recombination in yeast. Genetics 127:39-51.

37. Symington, L. S., and T. D. Petes. 1988. Expansions and contractions of the genetic map relative to the physical map of yeast chromosome III. Mol. Cell. Biol. 8:595-604.

38. Szostak, J. W., T. L. Orr-Weaver, R. J. Rothstein, and F. W. Stahl. 1983. The double-strand-break repair model for recombination. Cell 33:25-35.

39. Wagner, R., and M. Meselson. 1976. Repair tracts in mismatched DNA heteroduplexes. Proc. Natl. Acad. Sci. USA 73:4135-4139.

40. White, J. H., J. F. DiMartino, R. W. Anderson, K. Lusnak, D. Hilbert, and S. Fogel. 1988. A DNA sequence conferring high postmeiotic segregation frequency to heterozygous deletions in Saccharomyces cerevisiae is related to sequences associated with eucaryotic recombination hotspots. Mol. Cell. Biol. 8:1253-1258.

41. White, J. H., K. Lusnak, and S. Fogel. 1985. Mismatch-specific post-meiotic segregation frequency in yeast suggests a heteroduplex recombination intermediate. Nature (London) 315:350352.

42. Williamson, M. S., J. C. Game, and S. Fogel. 1985. Meiotic gene conversion mutants in Saccharomyces cerevisiae. I. Isolation and characterization of pms 1-1 and pms1-2. Genetics 110:609646.

43. Winston, F., F. Chumley, and G. R. Fink. 1983. Eviction and transplacement of mutant genes in yeast. Methods Enzymol. 101:211-228. 